\title{
Prostatectomía radical, radioterapia y vigilancia activa sin diferencias estadísticamente significativas en los resultados de mortalidad de pacientes con cáncer de próstata localizado
}

\section{Radical prostatectomy, radiation therapy and active surveillance with no statistically significant difference in mortality outcomes of patients with localized prostate cancer}

Abraham Orozco Martínez, Amilcar Aguayo Chávez
Recibido: octubre 2016

Aceptado: febrero 2017

Médico Pasante del Servicio Social. Instituto Mexicano del Seguro Social. Unidad de investigación en epidemiología clínica, Colima, Col.

Correspondencia: Abraham Orozco Martínez, abe_oreo@hotmail.com Amilcar Aguayo Chávez amilcaracha@gmail.com

En agradecimiento al Dr. José Guzmán Esquivel y al Dr. Benjamín Trujillo por aceptarnos dentro de su unidad de investigación y darnos la oportunidad de formar parte de ella.

El cáncer de próstata es uno de los principales problemas de salud pública en la población adulta y la neoplasia más frecuente en hombres mayores de 40 años de edad. La Agencia Internacional de Investigación del Cáncer (GLOBOCAN) señala que la incidencia más alta se registra en Austria y América del Norte (111.6 y 97.2 casos por cada 100,000 hombres, respectivamente) y la más baja en los países asiáticos (4.5 a 10.5 casos por 100,000 hombres). En
África del Sur y el Caribe las tasas de mortalidad son más altas en poblaciones afrodescendientes (19 a 24 muertes por cada 100,000 hombres). ${ }^{1}$

La mayoría de los pacientes con diagnóstico de cáncer de próstata fallece por causas ajenas a esta enfermedad. Un estudio efectuado en autopsias sugirió que $30 \%$ de los hombres mayores de 50 años y $70 \%$ de los mayores de 70 años de edad padece cáncer de próstata oculto. ${ }^{2}$ 
Los datos más recientes publicados por el Instituto Nacional de Cancerología (INCan) indican una tasa de mortalidad de 13 por cada 100,000 hombres. Durante el 2013 se estimó una tasa de mortalidad de 6 por cada 100,000 hombres de 20 años de edad o mayores en el Instituto Mexicano de Seguro Social (IMSS). ${ }^{1}$

Los lineamientos de la Asociación Americana de Urología (AUA) recomiendan, para la evaluación inicial y tratamiento del paciente con cáncer de próstata, tomar en cuenta los siguientes factores: 1) expectativa de vida del paciente (determinada por la edad y comorbilidades) y 2) características del tumor.

La decisión del tratamiento implica la participación del paciente, pues deben considerarse las complicaciones, efectos adversos y eficacia relativa del tratamiento, además de la calidad de vida, esta última de particular interés para cada caso. ${ }^{3}$

Hamdy y su grupo 4 realizaron un ensayo clínico aleatorizado, entre 1999 y 2009, en 82,429 hombres a quienes se les aplicó la prueba de antígeno prostático específico. Al final del reclutamiento se registraron 2,664 pacientes con diagnóstico de cáncer de próstata localizado, pero sólo 1,643 aceptaron someterse a una de tres modalidades de estudio, divididos al azar en: vigilancia activa (545 casos), cirugía (553 casos) o radioterapia (545). La edad media de los participantes fue de 62 años (límites de 50 a 69 años); la concentración media del antígeno prostático específico fue de $4.6 \mathrm{ng} / \mathrm{mL}$, con valores de 3.0 a $19.9 \mathrm{ng} / \mathrm{mL}$; el $77 \%$ de los pacientes tuvo tumores con puntuación de Gleason de 6 y $76 \%$ se encontraron en estadio T1c de la enfermedad. Estos resultados muestran que la mediana de 10 años se asoció con baja incidencia de mortalidad por cáncer de próstata (17 muertes en total), independientemente del tratamiento asignado, sin encontrar diferencias significativas ( $p=0.48)$. La cirugía y la radioterapia se asociaron con menor incidencia de evolución y metástasis de la enfermedad, comparada con la vigilancia activa (33 casos). ${ }^{4}$

Este estudio fue diseñado para evaluar la efectividad de los tres tratamientos actuales más importantes, enfocados en reducir la mortalidad del cáncer de próstata y evolución clínica de pacientes con enfermedad clínicamente localizada, mediante la determinación de antígeno prostático específico. Durante el estudio se observó que la evolución a metástasis de pacientes asignados a tratamiento quirúrgico o radioterapia fue menor que en quienes se mantuvieron en vigilancia activa. Estas diferencias mostraron que la terapia radical inmediata fue más efectiva que la vigilancia activa, pero no hubo una diferencia significativa en la mortalidad específica por la enfermedad u otras causas.

En México la esperanza de vida en hombres es de 72.3 años $^{3}$ y la manifestación del cáncer de próstata ocurre en sujetos mayores de 60 años de edad; sin embargo, el grupo de 75 años o mayores concentra casi la mitad de los casos. ${ }^{3}$ La Guía de Práctica Clínica del Cáncer de Próstata refiere que los pacientes asignados a tratamiento expectante o vigilancia activa muestran supervivencia a 10 años con calidad de vida adecuada, similar a la de quienes se operan para prostatectomía radical o radioterapia, por lo que se considera una opción viable en sujetos con neoplasias de bajo riesgo o esperanza de vida corta.

La vigilancia activa incluye la determinación de antígeno prostático específico cada 3 meses, tacto rectal cada 6 meses y obtención de biopsias prostáticas anualmente. Las ventajas de la vigilancia activa incluyen: 1) evita los efectos adversos de la terapia definitiva (algunas veces innecesaria); 2) no altera la calidad de vida y permite efectuar las actividades cotidianas del paciente; 3) las neoplasias pequeñas indolentes no reciben tratamientos innecesarios; y 4) disminuye costos iniciales. ${ }^{5}$ 
El tratamiento conservador es una estrategia apropiada en pacientes con pronóstico de vida menor de 10 años y en sujetos sanos de 65 años o mayores, a quienes se les considera de bajo grado de cáncer de próstata (Gleason menor a 7), afección menor de 50\% de cualquier núcleo y densidad del antígeno prostático específico (concentración del antígeno dividido entre el volumen prostático) menor de $0.15 .{ }^{6}$ Las desventajas de esta última incluyen pérdida de oportunidad para la cura, evolución del cáncer antes de finalizar el tratamiento, esquema a largo plazo de una neoplasia más agresiva, incluso más intensa y con mayores efectos adversos. La neuropreservación en la prostatectomía subsiguiente puede ser más difícil, lo que disminuye la oportunidad de preservar la función sexual después de la cirugía, además de incrementar la supervivencia con un cáncer no tratado y propiciar consultas médicas frecuentes. ${ }^{5}$

La vigilancia expectante, previa a la era de la medición del antígeno prostático específico, se relacionó con elevada mortalidad de cáncer de próstata, además de fallecimiento por causas ajenas a la enfermedad y metástasis, comparada con la prostatectomía radical ( 1 de cada 3 pacientes del grupo de vigilancia expectante falleció versus 1 de cada 5 del grupo de prostatectomía radical). ${ }^{7}$

La prostatectomía radical puede reservarse en pacientes con pronóstico de vida de 10 años o más, con enfermedad localmente avanzada, de bajo grado y concentraciones séricas de antígeno prostático específico menor de $20 \mathrm{ng} / \mathrm{mL}$, es decir, bien o moderadamente diferenciado (Gleason igual o menor de 7). ${ }^{5}$ La principal ventaja de la prostatectomía radical se basa en que no hay mejor manera de curar el cáncer si éste se encuentra completamente confinado en la próstata que la remoción quirúrgica. ${ }^{8}$

La radioterapia externa es una de las principales opciones de tratamiento para el cáncer de prós- tata clínicamente localizado; ofrece los mismos resultados de supervivencia a largo plazo que la cirugía, al igual que supervivencia libre de progresión de la enfermedad en pacientes de bajo riesgo. ${ }^{5}$

Las ventajas de la radioterapia son: procedimiento no invasivo, o mínimamente invasivo, y se relaciona con menos complicaciones (específicamente incontinencia urinaria), que ciertamente aparecen en pacientes sometidos a prostatectomía radical. ${ }^{9}$

Un estudio señaló que los pacientes sometidos a prostatectomía radical son más propensos a sufrir incontinencia urinaria, disfunción eréctil y urgencia miccional que quienes se someten a radioterapia después de 2 y 5 años de tratamiento. Sin embargo, no se observan diferencias significativas en la posibilidad de padecer complicaciones funcionales a 15 años (en los grupos estudiados apareció de igual manera). ${ }^{9}$

Hasta la fecha no se han encontrado diferencias estadísticamente significativas en la mortalidad relacionada con el tipo de tratamiento implementado en pacientes con cáncer de próstata, ya sea prostatectomía radical, radioterapia o vigilancia activa. La vigilancia activa debe considerarse en cualquier paciente con esperanza de vida promedio, cuya expectativa de vida sea menor de 5 años o con comorbilidades asociadas, pues someterlos a prostatectomía o radioterapia puede reducir, aún más, su calidad de vida y aumentar la tasa de mortalidad. De igual manera, el tratamiento conservador está contraindicado en pacientes menores de 65 años de edad, debido a la posibilidad de evolución de la enfermedad o metástasis y, por ende, por ende, se reduzcan las posibilidades de tratar y limitar el cáncer. Por lo mismo, no existe diferencia significativa en la mortalidad relacionada directamente con el cáncer de próstata entre el tratamiento quirúrgico y la radioterapia, por lo que la elección de ambos 
tratamientos dependerá de la calidad de vida del paciente, sin temor a seleccionar un tratamiento menos efectivo que otro.

\section{REFERENCIAS}

1. Instituto Mexicano del Seguro Social. Cáncer de próstata. México: [Sede web] IMSS. [en línea]. Dirección URL: $<$ http://www.imss.gob.mx/salud-en-linea/cancer-prostata>. [Consulta: 26 de septiembre 2016].

2. Coley CM, Barry MJ, Fleming C, Mulley AG. Early detection of prostate cancer. I. Prior probability and effectiveness of tests. Ann Intern Med1997;126:394-406.

3. Society American Cancer. Cáncer de próstata. [en línea]. Dirección URL: <http://www.cancer.org/acs/groups/cid/ documents/webcontent/002319-pdf.pdf>. [Consulta: 1 de septiembre 2016].

4. Hamdy FC, Donovan JL, Lane JA, Mason M, Metcalfe C, Holding $\mathrm{P}$, et al. 10-Year Outcomes after monitoring, surgery, or radiotherapy for localized prostate cancer. $\mathrm{N}$ Engl
J Med. en línea]. Dirección URL: <http://www.nejm.org/ doi/10.1056/NEJMoa1606220>.

5. Guía de Práctica Clínica Evidencias y Recomendaciones. Diagnóstico y tratamiento del cáncer de próstata en segundo y tercer nivel de atención. 2009. en línea]. Dirección URL: <http://www.cenetec.salud.gob.mx/descargas/gpc/ CatalogoMaestro/140_GPC_CA_PROSTATA/Gpc_ca_prostata.pdf>. [Consulta: 2 de octubre 2016].

6. Epstein JI, Chan DW, Sokoll LJ, et al. Non palpable stage T1C prostate cancer: prediction of insignificant disease using free/total prostate specific antigen levels and needle biopsy findings. J Urol 1998;160:2407-2411.

7. Bill-Axelson A, Holmberg L, Garmo H, Rider JR, Taari K, Busch C, et al. Radical Prostatectomy or Watchful Waiting in Early Prostate Cancer. N EngI J Med 2014;370(10):932-42.

8. Resnick MJ, Koyama T, Fan K-H, et al. Long-Term Functional Outcomes after Treatment for Localized Prostate Cancer. N Engl J Med. 2013;368(5):436-45. doi: 10.1056/ NEJMoa1209978.

9. Walsh PC, DeWeese TL, Eisenberger MA. Clinical practice. Localized prostate cancer. N EngI J Med 2007;357(26):2696705.

\section{AVISO PARA LOS AUTORES}

Revista Mexicana de Urología tiene una nueva plataforma de gestión para envío de artículos. En: https://www.revisionporpares.com/index.php/RMUrol podrá inscribirse en nuestra base de datos administrada por el sistema Open Journal System (OJS) que ofrece las siguientes ventajas para los autores:

- Subir sus artículos directamente al sistema.

- Conocer, en cualquier momento, el estado de los artículos enviados, es decir, si ya fueron asignados a un revisor, aceptados con o sin cambios, o rechazados.

- Participar en el proceso editorial corrigiendo y modificando sus artículos hasta su aceptación final. 